Génét. Sél. Evol., 1984, 16 (4), 417-430

\title{
Analyse expérimentale de trois niveaux d'interactions entre Drosophila melanogaster et le parasite Leptopilina boulardi (sympatrie, allopatrie, xénopatrie)
}

\author{
Y. CARTON \\ avec la collaboration technique de F. FREY \\ Laboratoire de Biologie et Génétique évolutives \\ C.N.R.S., F 91190 Gif-sur-Yvette
}

\begin{abstract}
Résumé
Si d'un point de vue théorique on conçoit aisément que pour 2 organismes vivant en étroite interdépendance, chaque modification chez l'un entraîne un changement évolutif chez l'autre et réciproquement, les preuves expérimentales d'un tel processus sont encore peu nombreuses. L'existence d'un parasite cosmopolite (Leptopilina boulardi) et spécifique de Drosophila melanogaster a permis d'analyser plusieurs paliers évolutifs correspondant à des temps de contact variables entre les 2 partenaires. Pour les infestations xénopatriques (les souches de $D$. melanogaster proviennent de régions où le parasite n'existe pas), la réussite parasitaire est faible avec une forte mortalité pupale. Pour les infestations allopatriques (les souches de l'hôte et du parasite proviennent de régions différentes), la réussite parasitaire est faible avec une forte mortalité larvaire. Pour les infestations sympatriques (les souches de l'hôte et du parasite proviennent de la même localité), la réussite parasitaire est élevée avec une faible mortalité. Ainsi les génomes de $D$. melanogaster peuvent être à des états d'évolution différents. La comparaison (par infestations de mêmes souches allopatriques de l'hôte) de 2 génomes du parasite révèle qu'ils sont plus ou moins bien adaptés à l'hôte, une souche insulaire d'introduction récente ayant une moins bonne réussite parasitaire. Ce sont les génomes qui ont interagi sur la plus longue période qui semblent être les plus compatibles. Dans les différents cas de sympatrie étudiés, l'homogénéité des résultats tend à prouver que le système a atteint un certain état d'équilibre qui apparaît plus favorable au parasite, D. melanogaster n'ayant développé aucune réaction de rejet cellulaire du parasite (par encapsulation).
\end{abstract}

Mots clés : Coévolution, Drosophile, parasite entomophage.

\section{Summary}

Experimental analysis of interactions between Drosophila melanogaster and parasitic wasp : Leptopilina boulardi (sympatry, allopatry, xenopatry)

Host parasite relationships offer a classical example of coevolution. Theory predicts that the host genotype is continually selected for an increased resistance while parasite genotype is selected for an increased virulence. Such interactions should play a significant role on the population dynamics of the 2 species and also on their capacity for evolution. Although convenient models in which 
both host and parasite are accessible to genetic analysis are rare, one such example is that of D. melanogaster and its parasitic wasp Leptopilina boulardi. In laboratory experiments, the relative success of parasitism was measured using 3 parameters : rate of successful parasitism (nRSP), rate of host emergence (nRHE) and rate of host mortality (nRM), with nRSP + nRHE + nRM $=100 \mathrm{p}$. 100 . Using Drosophila laboratory strains it was possible to show that the host genotype determines the relative success of infestation by a given strain of parasite. Since both Drosophila and Leptopilina are cosmopolitan species it was also possible to study the genetic coadaptation of sympatric populations and to compare the results obtained in « allopatric » infestations. In sympatric infestations (5 cases studied) nRSP was high and nRM very low; on the other hand, in allopatric infestations ( 8 cases studied) nRSP was low and nRM high, especially in larval mortality. In a 3rd type named «xenopatric » infestation (strain of D. melanogaster originating from localities where parasite is absent) nRSP was low and nRM high, but in this case we mainly observed a delayed mortality i.e. a pupal mortality. In « allopatric » infestations it was also possible to highlight genetic differences between 2 wasp populations. Since resultats obtained for sympatric infestation ( 5 cases studied) are very similar, we may-suppose that interactions between host and parasite are stabilized. However, in this coevolutionary process the genetic "victory " belongs to the parasite since the host is unable to maintain defence reactions against it.

Key words : Coevolution, Drosophila, parasitic wasp.

\section{Introduction}

On reconnaît généralement dans les associations plante-herbivore, proie-prédateur ou hôte-parasite l'existence d'interactions conduisant à une adaptation réciproque des génomes des 2 partenaires (BARBAUlt, 1981 ; LACHAISE, 1982). Toutefois les mécanismes mis en jeu sont encore mal élucidés. Roughgarden (1976) et FutuYma (1979) définissent la coévolution comme un processus biologique où la valeur adaptative des divers génotypes d'une espèce dépend, d'une part de leur composition génétique et de leur densité et, d'autre part, de ceux de l'espèce concurrente. JANZEN (1980) considère la coévolution comme un processus dans lequel tout changement génétique au niveau d'un caractère chez une espèce est la conséquence d'une modification génétique chez l'autre espèce qui se modifiera alors à son tour, le processus évoluant par interaction en cascade. On peut supposer, en effet, qu'il intervient assez de changements dans la structure génétique d'une espèce (sous l'influence du milieu) pour que de telles coactions soient générées continuellement. JANZEN (1980) précise toutefois que l'on fait un usage abusif de ce terme (PIANKA, 1978) lorsqu'on reconnaît obligatoirement un processus coévolutif dans tout phénomène de symbiose, de mutualisme, de prédation ou de parasitisme. Deux espèces peuvent apparaître comme parfaitement coadaptées sans que cela résulte nécessairement d'un phénomène coévolutif.

Le processus coévolutif reste difficile à mettre en évidence et se prête mal à l'expérimentation (BARBAULT, 1981). Les exemples les plus démonstratifs concernent les associations plante-phytophage telles que Ficus-Agaonidae (WIEBES, 1979), PassifloraHeliconius (GILBERT, 1971), blé-Mayetiola (HATChETT \& GAllun, 1970) et lin-rouille du lin (FLOR, 1971). Pour les 2 derniers cas, on a reconnu une situation telle qu'à un gène de résistance chez l'hôte correspond un gène de virulence chez le parasite. Ainsi, le concept coévolutif du "gene-for-gene » (PERSON, 1959) apparaît avoir effectivement une réalité génétique.

A partir de cette hypothèse, les auteurs ont aussi cherché, très souvent de façon spéculative, à savoir vers quel équilibre tend ce système. On suppose en général, si l'équilibre est stable, que pour tout accroissement de la résistance de l'hôte correspond 
une augmentation de la virulence du parasite. On assisterait donc à une escalade continue dans laquelle aucun des partenaires ne serait capable de supplanter l'autre ; c'est l'hypothèse de RosenzweIG (1975). VAN VALEN (1973) a proposé toutefois une autre hypothèse, prenant en compte la notion du taux constant d'extinction des espèces. L'environnement pour toute espèce se dégraderait continuellement à cause de la présence de compétiteurs et de prédateurs. L'espèce considérée comme proie n'échapperait alors à l'extinction qu'en compensant en permanence ce désavantage. Ainsi, les progrès adaptatifs d'une espèce ne seraient qu'apparents, à cause de l'abaissement de la valeur adaptative de l'autre espèce. Une autre possibilité consiste en des cycles alternés de la résistance de l'hôte et de la virulence du parasite maintenant la pérennité de l'association. Cette hypothèse a surtout fait l'objet de modélisations (YU, 1972 ; WILCox \& MACCLUER, 1979). Enfin, une dernière possibilité, d'ailleurs tout aussi plausible (DAY, 1974 ; FUTUYMA, 1979) consiste en ce que le système tende vers une moindre résistance de l'hôte et vers une moindre virulence du parasite, ceci conduisant à améliorer la compatibilité du système dans son ensemble et donc là encore sa pérennité.

Les parasites entomophages représentent un matériel favorable pour l'analyse du processus coévolutif. Dans la nature, l'introduction d'un parasite, dans un but de lutte biologique, a souvent permis de suivre son évolution génétique et celle du ravageur que l'on veut combattre.

MORRIS (1976) a démontré que les populations du lépidoptère Hyphantria ont une constitution génétique telle que leur aptitude à détruire l'œuf du parasite varie d'une année à l'autre. MULDREw (1953) au Canada, dans un exemple devenu classique, a montré que les populations d'une tenthrède qui attaque le bouleau sont devenues résistantes, 30 ans après l'introduction d'un parasite, ce qui se traduit par une nette augmentation du taux de capsules hémocytaires formées autour des œufs du parasite. Parallèlement, le parasite a évolué vers plus d'efficacité, les taux d'infestation rencontrés dans la nature passant de 19 p. 100 à 88 p. 100. En Californie, SALT \& VAN DEN BOSCH (1967) ont pu montrer au contraire que la population méridionale de Bathyplectes (Ichneumonidae) s'est, dans un laps de temps de 15 ans, modifiée dans le sens d'une meilleure adaptation à son hôte (coléoptère), le niveau de la réaction d'encapsulation passant de 50 p. 100 à 5 p. 100 chez ce dernier. WALKER (1959) a découvert que des populations du parasite Leptopilina heterotoma (= Pseudeucoila bochei) (hyménoptère Cynipidae) d'origines géographiques différentes présentaient des niveaux différents d'adéquation avec l'hôte Drosophila melanogaster.

Il est aussi possible au laboratoire de suivre l'évolution d'un tel système en maintenant en compétition sur plusieurs générations (par exemple en cage à populations) les 2 espèces. Olson \& Pimentel (1974) ont montré que lá mouche Musca, exposée pendant 40 générations au parasite Nasonia (hyménoptère Pteromalidae), développe une résistance telle que la fertilité du parasite est nettement diminuée.

Ce problème a été repris avec une nouvelle approche expérimentale, en bénéficiant d'un modèle favorable dans la nature, comme le préconisaient déjà BARTLETT \& BALL en 1966. Nous disposions avec le parasite Leptopilina boulardi (NORDLANDER, 1980) d'une espèce à spécificité stricte, très inféodée à Drosophila melanogaster (CARTON \& KITANO, 1981) et à distribution cosmopolite (CARTON \& ClARET, 1982). La femelle du parasite pond généralement ses œufs dans la cavité générale du $2^{\mathrm{e}}$ stade larvaire de la drosophile. Celle-ci continue son développement jusqu'au stade pupe. Le parasite se développe alors rapidement aux dépens de la pupe qui est entièrement détruite. C'est donc un parasite adulte qui sort du puparium (CARTON et al., 1985). PRICE (1980) a judicieusement 
souligné que si un parasite attaque plusieurs espèces, la tendance coadaptative entre ce parasite et l'un des hôtes sera certainement beaucoup moins affirmée. Avec ce matériel à large répartition, des situations écologiques fort différentes ont pu être retenues. Les populations de l'hôte et du parasite peuvent provenir de la même localité (sympatrie) ou de 2 localités très éloignées (allopatrie). Dans ce dernier cas, les 2 génomes n'ont jamais été en contact, même si l'hôte de cette région est infesté par le parasite. Une autre situation peut être retenue avec une population de $D$. melanogaster originaire d'une région où n'existe pas le parasite ; dans ce cas le terme de "xénopatrie " est proposé. Des infestations expérimentales réalisées au laboratoire dans ces différentes conditions nous ont permis d'évaluer plusieurs paramètres biologiques chez l'hôte et le parasite qui ont très certainement une dimension évolutive, c'est-à-dire sur lesquels a porté la sélection par l'autre partenaire.

\section{Matériel et méthodes}

\section{A. Origine des souches}

Les souches de D. melanogaster Meigen (1830) et de Leptopilina boulardi, Barbotin, Carton \& Kelner Pillault (1979) (n.c., NoRDLANDER, 1980) proviennent des localités suivantes (l'année de capture est indiquée entre parenthèses) :

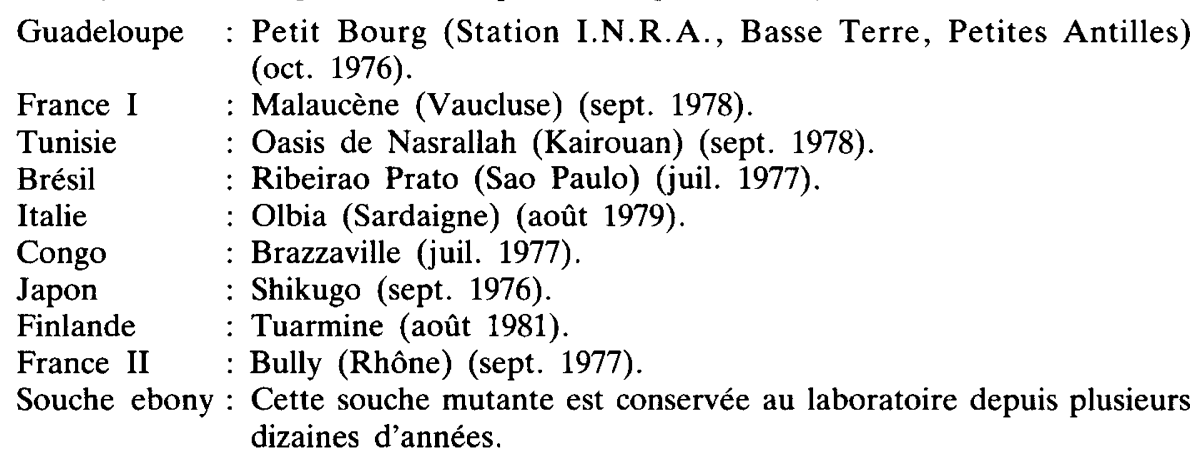

Ces 4 dernières souches proviennent de régions septentrionales où le parasite est absent.

Les souches de drosophiles sont maintenues en bouteilles (environ un millier d'individus sont repiqués à chaque génération). Les souches de parasite sont élevées en démomètres avec environ 2000 individus à chaque génération. Tous les 3 mois, 200 à 300 drosophiles élevées sans pression du parasite sont replacées dans le démomètre, afin qu'une sélection n'intervienne pas sur la population de l'hôte et donc du parasite.

\section{B. Protocole d'infestation}

La méthode expérimentale a été décrite en détail dans un travail précédent (CARTON \& Kitano, 1981) et sera résumée ici. 
40 larves $\mathrm{L}_{2}$ de $D$. melanogaster sont infestées pendant $24 \mathrm{~h}$ par 4 femelles de L. boulardi. Un lot de larves témoins (sans infestation) est aussi constitué. $24 \mathrm{~h}$ après la fin de l'infestation (fin de stade $\mathrm{L}_{3}$ ), les larves sont décomptées, permettant d'évaluer le taux de mortalité larvaire dans l'expérience (RLM) et dans le témoin $\left(\mathrm{RLM}_{\mathrm{c}}\right)$. Les larves ayant été soumises à l'infestation sont alors divisées en 2 lots : le premier lot est disséqué, permettant d'évaluer le degré d'infestation (DI). Le deuxième lot, ainsi que le témoin, est placé en élevage. 21 jours plus tard, on dénombre les drosophiles, les parasites et les pupes mortes, sans qu'il soit possible de déterminer l'origine de cette mortalité.

On peut ainsi évaluer le taux d'émergence de l'hôte (RHE), le taux de réussite parasitaire (RSP), le taux de mortalité pupale dans l'expérience (RPM) et dans le témoin $\left(\mathrm{RPM}_{\mathrm{c}}\right)$. Ces divers paramètres sont exprimés en pourcentage, par rapport au nombre de larves $\mathrm{L}_{3}$ mises en élevage.

L'expérience-type (2 lots de 40 larves pour l'expérimentation et 1 lot de larves témoins) a été renouvelée de 4 à 9 fois ; ainsi, 320 larves à 720 larves ont été soumises à l'infestation dans chaque type d'infestation (tabl. 1) à partir desquelles sont calculés les différents paramètres biologiques.

La comparaison des expériences nous imposait d'avoir des taux d'infestation équivalents. Comme nous l'avons montré (CARTON \& KITANO, 1981), un facteur de correction F permet de rapporter l'ensemble des résultats à un même niveau d'infestation $\left(\mathrm{DI}_{\mathrm{c}}\right)$. Il est préférable que la valeur retenue pour $\mathrm{DI}_{\mathrm{c}}$ corresponde à la moyenne des taux d'infestation relevés dans l'ensemble des expériences afin de minimiser l'erreur due à cette pondération :

$$
\mathrm{F}=\frac{\mathrm{DI}}{\mathrm{DI}}
$$

On peut alors calculer des valeurs pondérées pour chacun des paramètres : mortalité larvaire, mortalité pupale, mortalité totale, réussite parasitaire et émergence de l'hôte.

La mortalité larvaire pondérée (nRPM) est calculée de la façon suivante :

$$
\mathrm{nRLM}=\mathrm{F}\left(\mathrm{RLM}-\mathrm{RLM}_{\mathrm{c}}\right)
$$

La mortalité pupale pondérée (nRPM) est calculée de la façon suivante :

$$
\mathrm{nRPM}=\mathrm{F}\left(\mathrm{RPM}-\mathrm{RPM}_{\mathrm{c}}\right)
$$

La mortalité totale pondérée (nRM) prend comme valeur, après pondération : $n R M=F\left(R L M-R L M_{c}\right)+\left(100-F\left(R L M-R L M_{c}\right)\right) \times\left(F\left(R P M-R P M_{c}\right)\right)$

en se souvenant que :

$$
\mathrm{nRM}=\mathrm{nRLM}+(100-\mathrm{nRLM}) \times(\mathrm{nRPM})
$$

Le taux de réussite parasitaire après pondération (nRSP) est calculé de la façon suivante :

$$
\mathrm{nRSP}=\left[100-\mathrm{F}\left(\mathrm{RLM}-\mathrm{RLM}_{\mathrm{c}}\right)\right] \times[\mathrm{F}(\mathrm{RSP})]
$$

Le taux d'émergence de l'hôte (nRHE) après pondération est égal à :

$$
\mathrm{nRHE}=100-(\mathrm{nRM}+\mathrm{nRSP})
$$




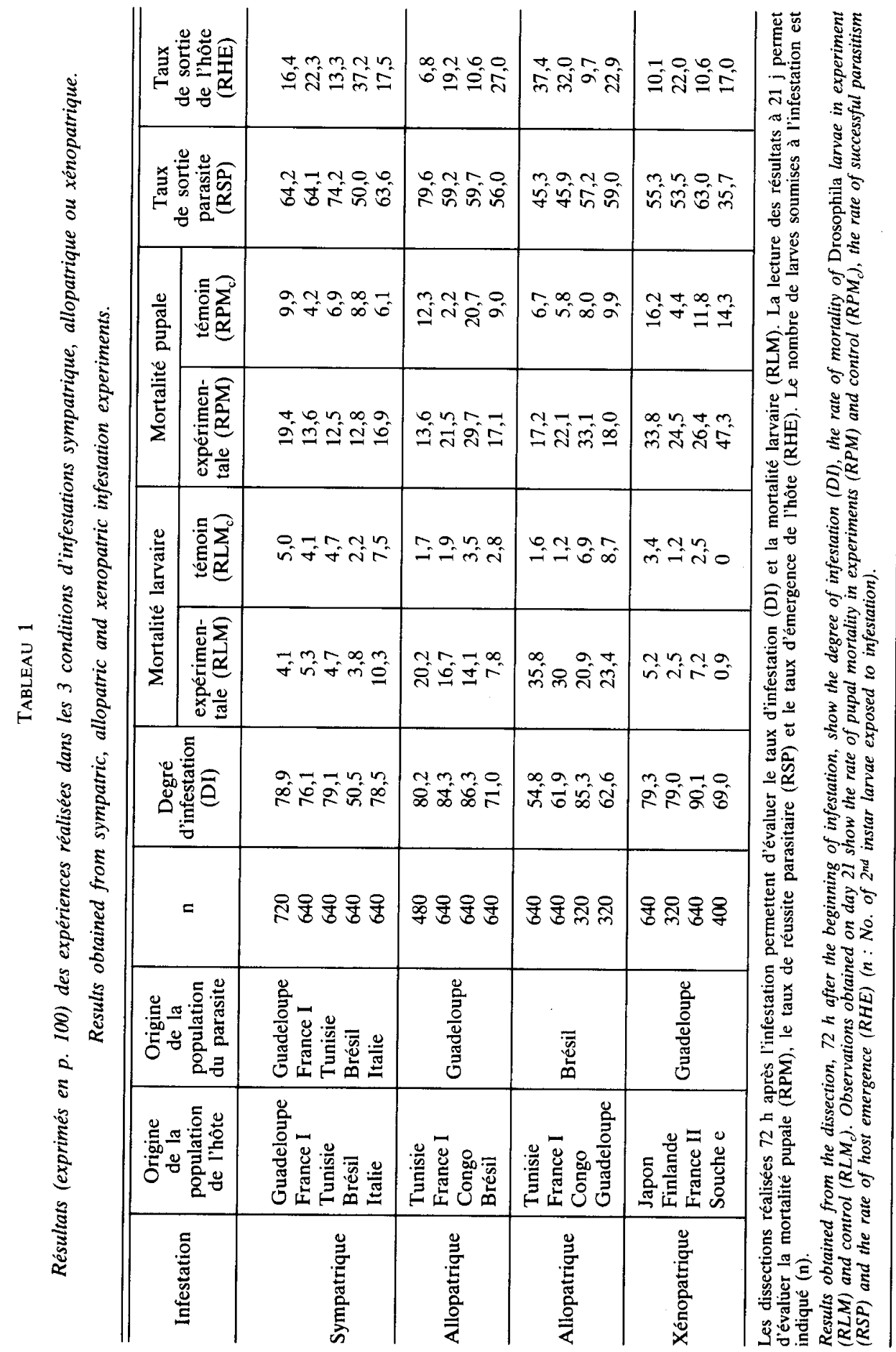


Les expériences ont été réalisées dans l'année qui suit la capture des souches (à l'exception des expériences réalisées avec la souche Shikugo qui a été réalisée deux ans après).

\section{Analyses statistiques des résultats}

Les tests non paramétriques permettent de connaître si 2 groupes indépendants de données proviennent de la même population sans que les conditions requises pour le test $\mathrm{t}$ de normalité et d'effectif soient remplies (cas des résultats exprimés en pourcentage). Le test de MANN WhITNEY, applicable au petit effectif (SIEGEL, 1956) a été appliqué à la comparaison de nos résultats.

Une analyse des correspondances (LEFEVRE, 1980) a été réalisée, permettant de prendre en compte l'ensemble des paramètres biologiques évalués dans l'expérimentation. Cette méthode présente aussi l'avantage de permettre la superposition des individus (le type d'infestation) et des variables (les paramètres biologiques) sur la même projection. Pour chaque type d'infestation, on a tracé des ellipses d'équiprobabilité à 75 p. 100 selon la méthode de HEALY (1972). La taille des ellipses renseigne sur la variabilité des résultats.

\section{Résultats}

Les résultats bruts des expériences sont présentés dans le tableau 1. Malgré toutes les précautions expérimentales, on constate que le taux d'infestation (DI) varie d'une expérience à l'autre entre 50,5 p. 100 et 90,1 p. 100 . La moyenne de l'ensemble (74,5 p. 100) a été utilisée pour effectuer une pondération (voir matériel et méthodes) permettant d'avoir le même degré d'infestation pour toutes les expériences (tabl. 2).

La validité d'une telle pondération, déjà justifiée dans un travail précédent (CARTON \& KITANO, 1981) peut être vérifiée. Il est possible d'estimer le niveau d'infestation par l'addition de la réussite parasitaire et de la mortalité (nRSP + nRM), sachant que cette mortalité est exclusivement imputable à l'infestation. Seule la mortalité liée au parasitisme est prise en compte dans ce paramètre (nRM) puisque la mortalité témoin, évaluée par les expériences sans infestation a été soustraite. En infestation sympatrique (tabl. 2), l'estimation nRSP + nRM $(73,5$ p. 100$)$ ne diffère pas du taux moyen d'infestation $(74,5$ p. 100$)$. Parallèlement, le paramètre nRHE présente des valeurs proches de 25,5 p. 100 , pourcentage correspondant aux larves non parasitées.

Dans les autres types d'infestation, les valeurs du paramètre nRHE s'écartent sensiblement de 25,5 p. 100 . Ces divergences peuvent s'interpréter par le fait que certaines larves parasitées peuvent survivre en éliminant l'œuf du parasite, ce qui se traduit par un nRHE supérieur à 25,5 p. 100. Dans le cas contraire (nRHE < 25,5 p. 100), on peut supposer que le dépôt de l'œuf n'a pas eu lieu dans toutes les larves piquées, cette piqûre entraînant toutefois la mort de la larve-hôte.

Pour chaque type d'infestation, il est possible d'évaluer le rendement de l'infestation parasitaire par le rapport :

$\frac{\mathrm{nRSP}}{\mathrm{DI}_{\mathrm{c}}} \quad$ (tabl. 2) 


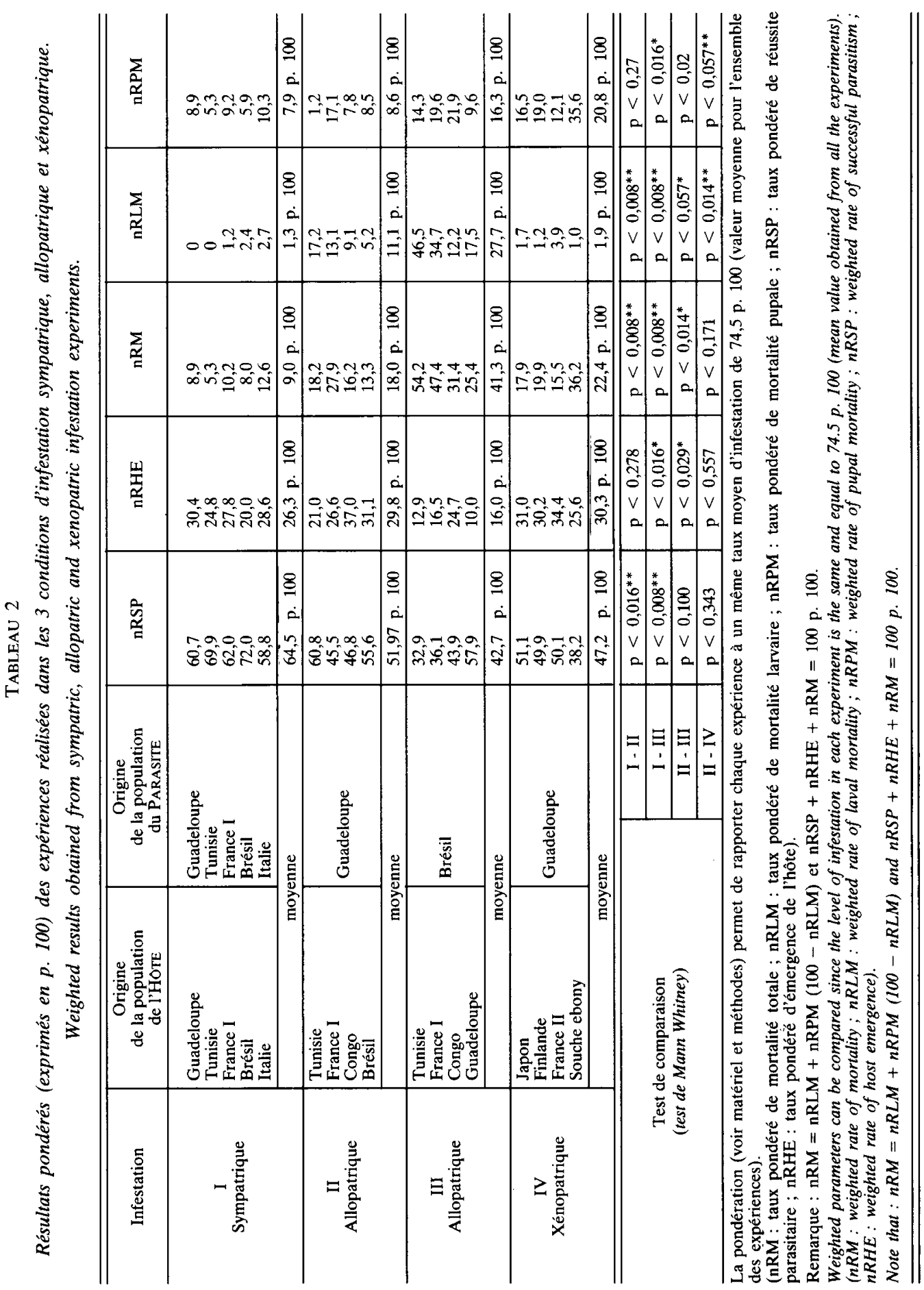


Ce paramètre permet d'apprécier le degré de compatibilité entre les génomes des 2 partenaires. On relève les valeurs suivantes :

- infestations sympatriques $\ldots \ldots \ldots \ldots \ldots \ldots \ldots \ldots \ldots$ 0,87

- infestations allopatriques : avec souche Guadeloupe du parasite .............. 0,70

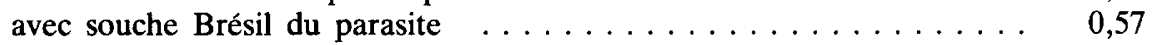

- infestations xénopatriques $\ldots \ldots \ldots \ldots \ldots \ldots \ldots \ldots \ldots$, $\ldots \ldots \ldots$

Dans le cas des 5 infestations sympatriques (tabl. 2) la réussite parasitaire est élevée (moyenne : 64,5 p. 100) alors que la mortalité totale reste faible $(9,0$ p. 100). Le rendement parasitaire est maximum $(0,87)$. D'autre part, les résultats apparaissent très homogènes (fig. 1).

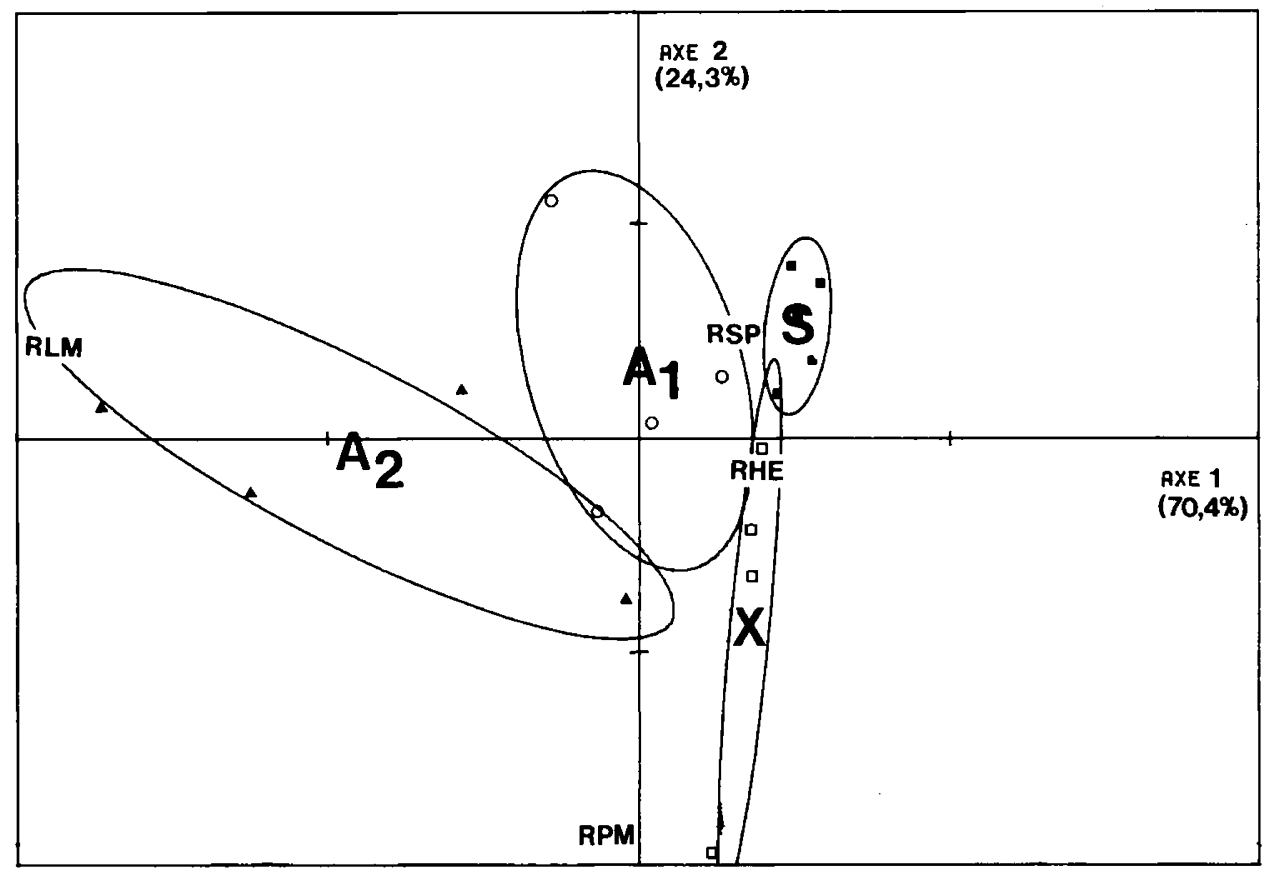

Fig. 1

Représentation graphique des résultats pondérés, obtenue par l'analyse multivariée des correspondances.

Correspondence analysis (multivariate analysis) of weighted results.

Chaque ellipse d'équiprobabilité (à 75 p. 100) correspond aux différents types d'infestation ( $S$ : sympatrie ; $A_{1}$ et $A_{2}$ : allopatrie ; $X$ : xénopatrie) (nRSP : taux pondéré de réussite parasitaire) ; nRHE : taux pondéré d'émergence de I'hôte ; nRLM et nRPM : taux pondéré de mortalités larvaire et pupale).

Analysis obtained for each type of infestation, specified by 75 p. 100 equal probability clouds ( $S$ : sympatric infestation; $A_{1}$ and $A_{2}$ : allopatric infestations; $X$ : xenopatric infestation) ( $n R S P$ : weighted rate of successful parasitism; $n R H E$ : weighted rate of host emergence; $n R L M$ and $n R P M$ : weighted rates of larval mortality and pupal mortality). 
Pour les 8 infestations allopatriques, les résultats obtenus avec les 2 souches géographiquement différentes du parasite, bien que plus marqués avec la population du Brésil, indiquent les mêmes tendances. La réussite parasitaire est significativement inférieure à celle observée dans les infestations sympatriques (51,9 p. 100 et 42,7 p. 100 au lieu de 64,5 p. 100). C'est toutefois au niveau de la mortalité que la différence est particulièrement nette : 18,9 p. 100 et 41,3 p. 100 contre 9,0 p. 100 . Cette mortalité apparaît se développer rapidement après l'infestation puisque c'est au stade larvaire que la mortalité est la plus forte $(11,1$ p. 100 et 27,7 p. 100$)$.

Dans le cas des infestations xénopatriques, nous pouvons les comparer à celles réalisées en allopatrie avec la même souche "Guadeloupe » du parasite. La réussite parasitaire est très comparable dans les 2 cas $(51,9$ p. 100 et 47,2 p. 100$)$ ainsi que la mortalité totale $(18,9$ p. 100 et 22,4 p. 100). Par contre, l'origine de cette mortalité est totalement différente : si en allopatrie la mortalité porte sur le stade larvaire, c'est essentiellement sur le stade pupe que porte la mortalité en infestation xénopatrique.

Si nous comparons entre elles les infestations réalisées en allopatrie avec les 2 souches géographiquement différentes de Leptopilina, nous observons que pour la souche Brésil le taux d'émergence de l'hôte est significativement plus faible (16 p. 100 contre 29,8 p. 100). De plus, la réussite parasitaire est moins élevée $(42,7$ p. 100 contre 52,0 p. 100), à la limite de la signification. Cette diminution dans les taux de développement de l'hôte et du parasite est due à une plus forte mortalité $(41,3$ p. 100 contre 18,9 p. 100) qui, dans les 2 cas, porte principalement sur le stade larvaire de l'hôte parasite.

Une analyse multidimensionnelle de ces résultats par la méthode de l'analyse des correspondances permet une représentation synthétique de ces résultats (fig. 1). L'axe 1 (70,4 p. 100 de l'information) discrimine principalement sur la mortalité larvaire, alors que l'axe 2 (24,3 p. 100 de l'information) discrimine sur la mortalité pupale et, dans une moindre mesure, sur la réussite parasitaire. Le taux d'émergence de l'hôte reste peu discriminant pour les 3 types d'infestation. Ainsi les infestations xénopatriques se caractérisent par une forte mortalité au stade pupale (ellipse très étirée suivant l'axe 2) alors que les infestations allopatriques réalisées avec la souche «Brésil 》du parasite (ellipse $\mathrm{A}_{2}$ ) présentent une forte mortalité larvaire (ellipse étirée suivant l'axe 1). Cette représentation permet aussi d'évaluer la variabilité dans chaque condition d'infestation. Cette variabilité étant proportionnelle à la taille de l'ellipse, c'est principalement pour les infestations allopatriques que celle-ci est la plus importante. Enfin, il est remarquable de noter que les résultats obtenus en xénopatrie ne s'éloignent pas nettement des autres types d'infestation.

\section{Conclusions}

Les différentes conditions expérimentales retenues ont permis de confronter des hôtes et des parasites dont les pools géniques ont eu ou non un passé en commun. Il était ainsi possible de vérifier si, au cours de la confrontation, les 2 partenaires sont devenus plus antagonistes ou plus compatibles ; ces 2 scénarios ont été proposés par les auteurs. Grâce à ces expériences, divers paliers évolutifs ont pu être analysés.

Il faut rappeler que le paramètre "mortalité » évalué dans ces expériences correspond à la mort du système biologique constitué par la larve de $D$. melanogaster et le parasite. Ce paramètre est un excellent indicateur du degré de compatibilité entre les génomes mis en présence. 
Les infestations xénopatriques correspondent à la situation qui se présente quand les 2 pools géniques sont confrontés pour la première fois. Ces conditions sont réalisées quand un parasite, de façon naturelle ou accidentelle, s'implante dans une nouvelle aire géographique. On note que la réussite parasitaire se situe à un niveau plus élevé qu'on pouvait le supposer. Cela correspond très certainement à l'existence d'une certaine compatibilité minimale entre 2 génomes, condition nécessaire pour qu'une association parasitaire puisse se réaliser entre 2 espèces. Aucune réaction de défense (de type cellulaire) contre le parasite n'est décelable, comme c'est le cas avec d'autres parasites (CARTON \& DAvid, 1983) ou d'autres espèces de drosophiles (CARTON \& KitANO, 1981). Une incompatibilité existe cependant, révélée par une forte mortalité qui intervient tardivement après la formation de la pupe, au moment où la larve de Leptopilina devient exoparasite et carnivore.

Pour les infestations allopatriques comparées aux précédentes, la réussite parasitaire augmente bien que la mortalité demeure élevée. Cette mortalité se développe toutefois très tôt, dès le stade larvaire de l'hôte. C'est la preuve d'une évolution de l'hôte qui devient capable de réagir très précocement contre le parasite. Cela conduit certes à la mort de l'hôte mais aussi du parasite ; on peut rapprocher cela du phénomène décrit comme « host suicide» par certains auteurs (SMITH TraIL, 1980).

En comparant les résultats en allopatrie à ceux obtenus en sympatrie, on a un moyen précis d'évaluer le niveau d'adaptation d'une certaine structure génique de l'hôte au génome sympatrique du parasite. En sympatrie, la mortalité a très fortement diminué, preuve d'une coadaptation spécifique des 2 partenaires. Chaque souche géographique de $D$. melanogaster n'est adaptée qu'à la souche sympatrique de $L^{\cdot}$. boulardi. Il apparaît donc qu'une longue période d'interactions entre les pools géniques des 2 populations puisse conduire à un certain état d'équilibre. Cette stabilité est confirmée par les résultats très homogènes obtenus dans les 5 cas étudiés. L'infestation a un rendement maximum $(0,87)$ comparée à celle observée en allopatrie $(0,70)$ ou en xénopatrie $(0,63)$, ceci pour la même souche de parasite (Guadeloupe). Les réactions d'incompatibilité sont considérablement réduites. Aucun mécanisme de défense cellulaire ne s'est développé chez aucune des populations de $D$. melanogaster étudiées, bien qu'elles soient très certainement soumises depuis une longue période à l'agression parasitaire. Ce sont donc les hypothèses de RosenzweIg (1973) et de DAY (1974) qui paraissent se vérifier. Le processus évolutif peut tendre, à condition que restent inchangées les pressions sélectives de l'environnement, vers un équilibre stable, où l'aptitude à se développer du parasite et la résistance de l'hôte sont fortement atténuées. C'est d'ailleurs une telle situation qui semble s'être développée après l'introduction du parasite Bathyplectes en Californie du Sud (SALT \& VAN DEN BosCH, 1967).

Un phénomène de coévolution suppose toutefois (JANZEN, 1980) un changement génétique chez les 2 partenaires. La discussion a porté jusqu'ici sur la comparaison des réponses de différentes populations de $D$. melanogaster confrontées à la même souche de parasite (Guadeloupe). La structure génétique de l'hôte quant à sa réceptivité au parasite apparaît différente, suivant qu'il a été soumis ou non (xénopatrie) à la pression du parasite (allopatrie et sympatrie). Qu'en est-il de l'évolution du génome du parasite ?

Les infestations réalisées en allopatrie avec 2 souches de parasite ayant un passé très différent permettent d'aborder cette question. La souche Brésil paraît être beaucoup moins bien acceptée que la souche Guadeloupe par les mêmes souches de $D$. melanogaster, la mortalité étant beaucoup plus élevée (41,3 p. 100 au lieu de 18,0 p. 100). Lè rendement parasitaire n'est que de 0,57 pour le parasite originaire du Brésil, alors qu'il est de 0,7 pour celui issu de Guadeloupe. On peut donc supposer que les génomes 
du Brésil sont plus étroitement adaptés à la population sympatrique de $D$. melanogaste que ne le sont ceux de Guadeloupe. C'est ce qui se vérifie pleinement puisque dan les infestations sympatriques c'est pour l'association du Brésil que l'on rencontre l meilleur rendement : 0,97. Au contraire, une adaptation plus faible du génome Guade loupe du parasite à la population sympatrique de l'hôte peut expliquer sa meilleur « acceptation " par les 4 souches allopatriques de drosophiles. Un argument biogéogra phique peut aussi être avancé : il est raisonnable de penser que $L$. boulardi est d'intro duction plus récente sur une île comme la Guadeloupe et que le temps de coévolutior entre les 2 génomes a été plus court.

En définitive, les génomes des 2 partenaires peuvent être à des états d'évolutior différents ; ce sont ceux qui ont interagi sur la plus longue période qui se révèlent les plus compatibles, c'est-à-dire les plus coadaptés.

Un processus sélectif en cascade a pu intervenir dans ce phénomène. Il existe, en effet, au sein d'une même population de $D$. melanogaster une variabilité génétique de sa réceptivité à $L$. boulardi (BouletreAu \& FouILlET, 1982), sur laquelle a pu jouer la sélection par le parasite.

Le parasite apparaît toutefois s'être beaucoup mieux adapté à l'hôte que ce dernier n'a pu le faire. L'absence de réaction de défense dans les infestations étudiées ici le confirme.

On pourrait donc supposer qu'aucun phénomène sélectif améliorant la capacité de défense de $D$. melanogaster ait pu agir, puisque toute larve parasitée (n'ayant pas réussi à détruire le parasite) ne donne aucune descendance. C'est une telle hypothèse qui est avancée par Bouletreau \& FouIllet (1982). Il existe en fait une autre possibilité.

Dans les espèces de Drosophila appartenant au groupe melanica parasitées par Leptopilina heterotoma les œufs du parasite n'éclosent pas ou, s'ils éclosent, les larves meurent rapidement sans qu'aucune réaction cellulaire ne soit décelable (NAPPI \& STREAMS, 1970 ; NAPPI, 1970). Un tel phénomène paraît exister dans les cas étudiés ici.

Dans les infestations allopatriques et xénopatriques réalisées avec la souche Guadeloupe du parasite, l'évaluation du niveau d'infestation (nRSP + nRM) donne des valeurs ( 70,9 p. 100 et 69,8 p. 100 respectivement) inférieures au taux moyen réel d'infestation $\left(\mathrm{DI}_{\mathrm{c}}=74,5\right.$ p. 100). Cela pourrait correspondre à des larves de drosophiles effectivement infestées mais chez qui le parasite ne s'est pas développé. Il existe donc là un processus sur lequel la sélection a pu jouer en faveur de l'hôte mais, réciproquement, auquel le parasite a pu échapper. Il est certain que dans cette étude l'influence du milieu n'a pu être prise en compte. Les populations que nous avons retenues proviennent de régions très différentes. Il est concevable que les facteurs environnementaux (rôle des facteurs climatiques sur la stabilité des effectifs, ressources alimentaires, pression des prédateurs et des autres parasites, ...) puissent aussi intervenir, variant énormément d'une région à l'autre.

Un dernier aspect mérite d'être souligné. C'est pour les infestations sympatriques (fig. 1) que la variabilité est la plus faible, ceci se traduisant par une ellipse d'équiprobabilité très réduite. Il est possible de suggérer que pour toute association s'étant réalisée sur une période de temps assez longue en des lieux différents, on arrive à une situation très comparable, représentant en quelque sorte un état d'équilibre stable. Le processus coévolutif aurait atteint un point d'équilibre. Cette situation correspondrait alors à celle proposée dans les hypothèses de VAN VALEN (1973) et de MAYNARD SMITH (1976).

Reçu le 20 décembre 1983. Accepté le 12 avril 1984. 


\section{Remerciements}

Nous tenons à remercier F. FREY de son aide pour la réalisation de ce long travail expérimental. J. DAvid a contribué par ses discussions à l'amélioration de ce manuscrit.

Ce travail est réalisé dans le cadre du contrat ATP «Biologie des populations » du C.N.R.S., de l'accord de coopération C.N.R.S.-N.S.F. (France-U.S.A.) et de l'accord de coopération C.N.R.S.-D.R.S.T. (France-Tunisie).

\section{Références bibliographiques}

Barbault R., 1981. Ecologie des populations et des peuplements. 200 pp., Masson, Paris.

Barbotin F., Carton Y., Kelner-Pillault S., 1979. Morphologie et biologie de Cothonaspis (Cothonaspis) boulardi n.sp., parasite de drosophiles. Bull. Soc. Entomol. Fr., 84, 20-26.

Bartlett B.R., Ball J.C., 1966. The evolution of host suitability in a polyphagous parasite with special reference to the role of parasite egg encapsulation. Ann. Entomol. Soc. Am., 59, 42-45.

Bouletreau M., Fouillet P., 1982. Variabilité génétique intrapopulation de l'aptitude de D. melanogaster à permettre le développement d'un hyménoptère parasite. C. R. Acad. Sci., Paris, 295, 775-778.

Carton Y., Claret J., 1982. Adaptative significance of a temperature induced diapause in a cosmopolitan parasitoïd of Drosophila. Ecol. Entomol., 7, 239-247.

CARTON J., DAvid J., 1983. Reduction of fitness in Drosophila adults surviving parasitization by a cynipid wasp. Experientia, 39, 231-233.

Carton Y., Kitano H., 1981. Evolutionary relationships to parasitism by seven species of the Drosophila melanogaster subgroup. Biol. J. Linn. Soc., 16, 227-241.

Carton Y., Bouletreau M., Van Alphen J., Van Lenteren J., 1985. The Drosophila parasitic wasps. In : Ashburner M., Thompson J. (ed.). The Genetics and Biology of Drosophila. Academic Press, New York (sous presse).

DAY P.R., 1974. Genetics of host parasite interaction. 238 pp., Freeman, San Francisco.

FLOR H.R., 1971. Current status of the gene-for-gene concept. Annu. Rev. Phytopathol., 9, 275-296.

FutuYma D.J., 1979. Evolutionary biology. 565 pp., Sinauer Associates, Sunderland, Massachusetts.

GILBERT L.E., 1971. Butterfly-plant coevolution: has Passiflora adenopoda won the selectional race with heliconiine butterflies? Science, 172, 585-586.

Hatchett J.H., Gallun, 1970. Genetics of the ability of the Hessian fly Mayetolia destructor to survive on wheats having different genes for resistance. Ann. Entomol. Soc. Am., 63, 1400-1407.

Healy M.J., 1972. Drawing a probability ellipse. J. R. Stat. Soc., 21, 202-204.

JANZEN D.H., 1980. When is it coevolution? Evolution, 34, 611-612.

LACHAISE D., 1982. Comment les peuplements de plantes et d'insectes phytophages se façonnent mutuellement : la théorie coévolutive de la structure des peuplements. Rev. Ecol. (Terre et Vie), 36, 481-537.

LEFEBVRE J., 1980. Introduction aux analyses statistiques multidimensionnelles. 259 pp., Masson, Paris.

Maynard-Smith J., 1976. A comment on the Red Queen. Am. Nat., 110, 325-330.

MORRIS R.F., 1976. Influence of genetic changes and other variables on the encapsulation of parasites by Hyphantria aunea. Can. Entomol., 108, 673-684.

MULDREw J.A., 1953. The natural immunity of the larch sawfly [Pritisphora erichsonii (NTG.)], to the introduced parasite Mesoleius tenthredinis Morley, in Manitoba and Saskatchewan. Can. J. Zool., 31, 167-312.

NAPPI A.J., 1970. Defense reactions of Drosophila euronotus larvae against the hymenopterous parasite Pseudeucoila bochei. J. Invertebr. Pathol., 16, 408-418.

NAPPI A.J., Streams F.A., 1970. Abortive development of the Cynipid parasite Pseudeucoila bochei (Hymenoptera) in species of the Drosophila melanica group. Ann. Entomol. Soc. Am., 63, 321-327. 
NORDLANDER G., 1980. Revision of the genus Leptopilina Forster, 1869, with notes on the status of some genera (Hymenoptera, Cynipoidea : Eucoilidae). Entomol. Scand., 11, 428-453.

Olson D., Pimentel D., 1974. Evolution of resistance in a host population to attacking parasite. Environ. Entomol., 3, 621-624.

PERSON C., 1959. Gene-for-gene relationships in host-parasite systems. Can. J. Bot., 37, 1101-1130. PIANKA E.R., 1978. Evolutionary ecology. 356 pp., Harper \& Row, New York.

PrICE P.W., 1980. Evolutionary biology of parasites. 237 pp., Princeton University Press.

RosenZWeig M.L., 1973. Evolution of the predator isocline. Evolution, 27, 84-94.

ROUGHGARDEN J., 1976. Resource partitioning among competing species, a coevolutionary approach. Theor. Popul. Biol., 9, 388-424.

Salt G., van DEN BosCh R., 1967. The defense reactions of three species of Hypera (Coleoptera Curculionidae) to an ichneumon wasp. J. Invertebr. Pathol., 9, 164-177.

SIEGEL S., 1956. Non parametric statistics for the behavioral sciences. 312 pp., Mac Graw Hill, New York.

SMITH-Trail B.R., 1980. Behavioral interactions between parasites and hosts : host suicide and the evolution of complex life cycles. Am. Nat., 116, 77.

VAN VAlen L., 1973. A new evolutionary law. Evol. Theory, 1, 1-30.

WALKER I., 1959. Die Abwehrreaktion des Wirtes Drosophila melanogaster gegen die zoophage Cynipide Pseudeucoila bochei Weld. Rev. Suisse Zool., 66, 569-632.

WiEbES J.T., 1979. Coevolution of figs and their insect pollinators. Ann. Rev. Ecol. Syst., 10, 1-12.

WilCoX D.L., MACCLUER J.W., 1979. Coevolution in predator prey systems : a saturation kinetic model. Am. Nat., 113, 163-183.

YU P., 1972. Some host parasite genetic interaction models. Theor. Popul. Biol., 3, 347-357. 